\title{
Effect Analysis of Time and Carrier Frequency Offset on the Performance of Distributed Transmit Beamforming for Emergency Radio
}

\author{
Ding YUAN, Houde QUAN, Huixian SUN \\ Dept. of Information Engineering, Mechanical Engineering College, Heping West Road No.97, 050003 Shijiazhuang, China \\ ydabroad@163.com \\ Submitted December 20, 2016 / Accepted February 20, 2017
}

\begin{abstract}
In emergency radio scenario, distributed transmit beamforming (DTBF) enables a set of distributed radio nodes to transmit cooperatively to get a better performance. DTBF performance has been analyzed for various node distributions in the literature. However, time offset (TO) and carrier frequency offset (CFO) may exist between transmitting nodes, due to each radio node is equipped with its own clock circuit and local oscillator. Effect analysis of TO and CFO on DTBF performance is an open issue. This paper evaluates the effect of TO and CFO on performance of DTBF for arbitrary node distributions. In this study, TO and CFO are converted into phase offset (PO), and non-parametric kernel method is used to calculate the PDFs of node and offsets. Theoretical analysis and simulation results show that TO and CFO result in degradation of the mainlobe power and affect the beampattern characteristics.
\end{abstract}

\section{Keywords}

Distributed transmit beamforming, emergency radio, time offset, carrier frequency offset, non-parametric kernel method

\section{Introduction}

Distributed transmit beamforming (DTBF) coordinates a set of transmitting nodes to form a virtual antenna array. All nodes transmit a common signal with designed beamforming weights so as to form a beam in the desired direction of transmission. This technique can translate the power gain of DTBF into increases in range, rate or energy efficiency [1]. In terms of interference suppression, DTBF can provide benefits by strengthening the desired signal in intended direction [2]. In tactical communication, the link between receiver and transmitter can be weak or even be interrupted when the receiver is out of communication range or suffers from interference. This scenario is categorized as emergency radio [3]. In this situation, the distributed single-antenna transmitting radio nodes can be cooperated to get beamforming gain by adopting DTBF technique. This cooperative approach with the framework of MISO is a complementarity to the existing communication mode.

DTBF performance has been analyzed in the exiting literature [4-6]. Most researches mainly cared about the node factors and their effects on beamforming, such as node distribution, node placement errors and the number of nodes. The characteristics of beampattern have been studied for Uniform, Gaussian, Uniform Circle and arbitrary node distributions in [7-9].

Considering DTBF in emergency radio situation, every radio node in distributed beamformer has its own clock and local oscillator. Time offset (TO) and carrier frequency offset (CFO) may exist between transmitting nodes, which also can affect DTBF performance. It is an open issue to evaluate the effects of time and carrier frequency offsets on DTBF with arbitrary node distribution. Inspired by [9] and [10], this paper presents performance analysis of DTBF with arbitrary node distribution in presence of TO and CFO. The basic idea of this paper is to convert time and carrier frequency offsets into corresponding phase offset (PO) and then analyze their effects on beampattern properties in statistical way. Node and offset distributions are modeled by using the non-parametric kernel method.

The remainder of this paper is organized as follows: Section 2 provides the system model and gives the expression of beampattern with arbitrary node distribution by using kernel method. Section 3 gives time offset and carrier frequency offset existing between transmit nodes, and analyzes their effects on beampattern. Section 4 shows the simulation results and offers discussion. Section 5 concludes this paper work.

\section{System Model and Average Beam- pattern}

\subsection{System Model}

The system model is illustrated in Fig. 1. Considering $N$ transmitting radio nodes are randomly deployed in a disk area of radius $R$ in the $(x, y)$ plane. The Cartesian coordinates of 


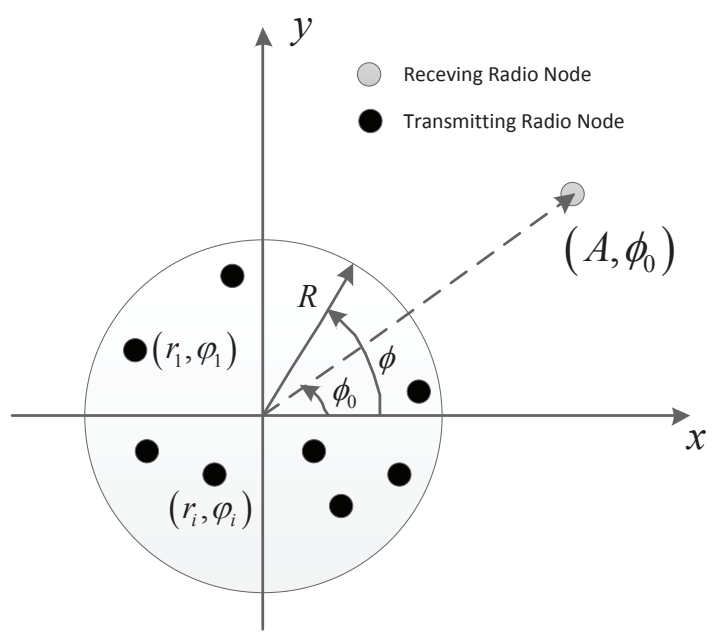

Fig. 1. System model.

node $i$ are $\left(x_{i}, y_{i}\right), i=1, \ldots, N$ and the corresponding spherical coordinates are $\left(r_{i}=\sqrt{x_{i}^{2}+y_{i}^{2}}, \varphi_{i}=\arctan \left(y_{i} / x_{i}\right)\right)$. Define $\mathbf{r}=\left[r_{1}, r_{2}, \cdots, r_{N}\right] \in[0, \mathbf{R}]$ and $\varphi=\left[\varphi_{1}, \varphi_{2}, \cdots, \varphi_{N}\right] \in$ $[-\pi, \pi)^{N}$. The receiving radio node is located at $\left(A, \phi_{0}\right)$. The goal of DTBF is to coordinate the transmitting radio nodes and collaboratively send a common message to the destination. Several system assumptions are made as following [4]:

- The radio nodes are assumed to be static over the communication period. And each radio node is equipped with a single isotropic antenna.

- All transmitting radio nodes are sufficiently separated and the mutual effects among antennas are not considered.

- The path losses between each transmitting node and the destination are identical. Signal reflection or scattering situations are neglected in this discussion.

- The transmitting nodes have got enough priori information, such as the accurate distance between each transmitting node and the destination, the elevation direction. All radio nodes share the same transmitting information in advance.

\subsection{Average Beampattern}

Beampattern describes the array gain in the whole region $\phi \in[-\pi, \pi)$. Here we focus on the radiation pattern in the far-field region, and the far-filed condition $A \gg r_{i}$ holds. The Euclidean distance between node $i$ and the location $(A, \phi)$ can be defined as:

$$
\begin{aligned}
d_{i}(\phi) & =\sqrt{A^{2}+r_{i}^{2}-2 A r_{i} \cos \left(\phi-\varphi_{i}\right)} \\
& \approx A-r_{i} \cos \left(\phi-\varphi_{i}\right)
\end{aligned}
$$

The initial phase of node $i$ is set to

$$
\Psi_{i}=-\frac{2 \pi}{\lambda} d_{i}\left(\phi_{0}\right)
$$

where $\lambda$ is the wavelength. This value is set to cancel out the channel effects.

The array factor is defined as:

$$
\begin{aligned}
F(\phi \mid \mathbf{r}, \varphi) & =\frac{1}{N} \sum_{i=1}^{N} \mathrm{e}^{\mathrm{j} \Psi_{i}} \mathrm{e}^{\mathrm{j} \frac{2 \pi}{\lambda} d_{i}(\phi)} \\
& =\frac{1}{N} \sum_{i=1}^{N} \mathrm{e}^{\mathrm{j} \frac{2 \pi}{\lambda}\left[d_{i}(\phi)-d_{i}\left(\phi_{0}\right)\right]} .
\end{aligned}
$$

Without loss of generality, we set $\phi_{0}=0$. Substituting (1) into (3), the array factor can be rewritten as:

$$
\begin{aligned}
F(\phi \mid \mathbf{r}, \varphi) & =\frac{1}{N} \sum_{i=1}^{N} \mathrm{e}^{\mathrm{j} \frac{2 \pi}{\lambda} r_{i}\left[\cos \left(\varphi_{i}\right)-\cos \left(\phi-\varphi_{i}\right)\right]} \\
& =\frac{1}{N} \sum_{i=1}^{N} \mathrm{e}^{-\mathrm{j} \frac{4 \pi}{\lambda} r_{i}\left[\sin \left(\varphi_{i}-\frac{\phi}{2}\right) \sin \frac{\phi}{2}\right]} \\
& =\frac{1}{N} \sum_{i=1}^{N} \mathrm{e}^{-\mathrm{j} a z_{i}}
\end{aligned}
$$

where $a=a(\phi)=4 \pi \sin \frac{\phi}{2}$ and $z_{i}=\frac{r_{i}}{\lambda} \sin \left(\varphi_{i}-\frac{\phi}{2}\right)$. Here $z_{i}$ is related to the node coordinates $\left(r_{i}, \varphi_{i}\right)$ and may have different probability density functions (PDFs) according to the deployment of radio nodes. Define $\mathbf{z}=\left[z_{1}, z_{2}, \cdots, z_{N}\right] \in(-\infty,+\infty)^{N}$, then $F(\phi \mid \mathbf{r}, \varphi)$ can be rewritten as $F(\phi \mid \mathbf{z})$.

The far-field beampattern can be defined as

$$
\begin{aligned}
P(\phi \mid \mathbf{z}) & =|F(\phi \mid \mathbf{z})|^{2} \\
& =F(\phi \mid \mathbf{z}) F^{*}(\phi \mid \mathbf{z}) \\
& =\frac{1}{N}+\frac{1}{N^{2}} \sum_{i=1}^{N} \mathrm{e}^{-\mathrm{j} a z_{i}} \sum_{l=1, l \neq i}^{N} \mathrm{e}^{\mathrm{j} a z_{l}} .
\end{aligned}
$$

As $P(\phi \mid \mathbf{z})$ is related to random variable $z_{i}$, the characteristics of beampatterns should be derived in statistical sense. The average beampattern is defined as:

$$
P_{\mathrm{av}}(\phi)=E_{\mathbf{z}}\{P(\phi \mid \mathbf{z})\}
$$

where $E_{x}\{\cdot\}$ represents expectation with respect to random variables $x$. As shown in (6), the PDF of $z_{i}$ is required in the calculation of beampattern, which can be estimated by using the non-parametric kernel method.

The non-parametric kernel method in [9], [10] can estimate the PDF of $z_{i}$ for radio nodes distributions with or without explicit PDFs. Its basic procedure is to get an estimated PDF from a survey set. Assuming that $M$ sample points are available for $z_{i}$, the estimated PDF can be obtained from survey set $\left\{\bar{z}_{1}, \bar{z}_{1}, \cdots, \bar{z}_{M}\right\}$ as:

$$
f_{z_{i}}(z)=\frac{1}{\sqrt{2 \pi} M h} \sum_{t=1}^{M} \exp \left(-\frac{\left(z-\bar{z}_{t}\right)^{2}}{2 h^{2}}\right)
$$

where $\exp (\cdot)$ is the Gaussian kernel function and $h$ is the width of kernel function. As for kernel estimate, the number of sample points, the kernel function and its width can affect the estimated results. These values should be chose 
according to the specific application. Here we use Gaussian kernel method as shown in (7). This choice has been proved suitable to the performance analysis in [10].

As detailed shown in [10], the average beampattern can be derived as:

$$
P_{\mathrm{av}}(\phi)=\frac{1}{N}+\left(1-\frac{1}{N}\right)\left|A_{z}\right|^{2}
$$

where $A_{z}=\frac{1}{M} \sum_{t=1}^{M} \mathrm{e}^{-\mathrm{j} a \bar{z}_{t}}$. The term $1 / N$ represents the average power level of the sidelobe, whereas the second term contributes to the mainlobe.

\section{The Average Beampattern with Time Offset and Carrier Frequency Offset}

Unlike traditional beamforming, when each of the array elements is controlled by a same source, coordinated nodes in distributed beamforming have their own sources [11]. Therefore, apart from node factors, we also need pay attention to these offsets between transmitting nodes, which can be treated as independent variables. Here we try to evaluate these offsets effects in statistical way. Non-parameter kernel method is adopted here to deal with offsets distributions with or without explicit PDFs.

\subsection{Time Offset}

Time offset includes the discrepancy caused by node clock oscillators and transmission latency. As for the effect analysis, we focus on the clock differences between transmission radio nodes. Three terms are used to describe the difference of running behavior of node clock [12]. Define $T(t)=t$ as the reference clock and $C_{i}(t)$ as the clock of radio node $i$, the following definitions are presents:

- Clock offset: the difference between $C_{i}(t)$ and $T(t)$, which is represented as:

$$
C_{i}(t)-T(t)=C_{i}(t)-t .
$$

The initial offset of node $i$ at time 0 is defined as $\beta_{i}=C_{i}(0)$.

- Clock skew: the difference between the first derivative of $C_{i}(t)$ and $T(t)$, which is also called frequency offset and is shown below:

$$
\frac{\partial C_{i}(t)}{\partial t}-\frac{\partial T(t)}{\partial t}=\frac{\partial C_{i}(t)}{\partial t}-1
$$

Here define $\alpha_{i}$ as the skew rate of the clock and $\alpha_{i}=\partial C_{i}(t) / \partial t$. The skew rate determines how much time will change over a given observation duration. As for $\alpha_{i},\left|\alpha_{i}-1\right| \leq \rho$ holds, where $\rho$ is the tolerance value in parts per million (ppm), which is determined by fabrication issues and climate conditions [13]. For a perfect clock, the skew rate is 1 .
- Clock drift: the difference between the second derivative of $C_{i}(t)$ and $T(t)$, which shows the time varying of clock skew. When the observation duration $T_{\text {od }}$ is small, the effects of clock drift can be neglected.

For a given observation duration $T_{\mathrm{od}}$, the TO of radio node $i$ can be represented as $\Delta t_{i}$. Assuming the time offset $\left\{\Delta t_{i}\right\}_{i=1, \cdots, N}$ are independent and identically distributed, we have:

$$
\Delta t_{i}=\left(\alpha_{i}-1\right) T_{\mathrm{od}}+\beta_{i}
$$

Moreover, $\alpha_{i}$ and $\beta_{i}$ are related to phase difference of node clock circuit in some research. The phase difference can be equivalent to time difference by multiplying a factor as $1 /\left(2 \pi f_{\text {clock }}\right)$, where $f_{\text {clock }}$ is the nominal frequency of the clock oscillator.

Here we convert TO to phase offset (PO) so as to analyze its influence and denote that:

$$
\Delta \varphi_{t i}=2 \pi f_{\mathrm{c}} \Delta t_{i}
$$

where $\Delta \varphi_{t i}$ represents the PO of radio node $i$ caused by corresponding offset $\Delta t_{i}$, and $f_{\mathrm{c}}$ is the radio carrier frequency.

\subsection{Carrier Frequency Offset}

It is assumed that all transmitting radio nodes are equipped with the same type of local oscillator to generate carrier signal. Due to the accuracy and stability of the oscillator, the cooperative nodes have different frequency deviations. Define $\Delta f_{i}$ as the frequency offset of node $i$, which represents the difference between the nominal carrier frequency $f_{\mathrm{c}}$ and actual frequency of node $i$.

Here $\left\{\Delta f_{i}\right\}_{i=1, \cdots, N}$ are also assumed to be independent and identically distributed. As for performance analysis, we adopt the PDF assumption in [3] and [14]. $\Delta f_{i}$ is treated as a random variable, and its mean and standard deviation of CFO are shown below:

$$
\begin{gathered}
E\left\{\Delta f_{i}\right\}=0, \\
\sigma_{\Delta f_{i}}=\sqrt{E\left\{\Delta f_{i}^{2}\right\}}=\alpha_{i} f_{\mathrm{c}}
\end{gathered}
$$

where $\alpha_{i}$ is the corresponding frequency skew rate of clock crystal in ppm.

For a given observation duration $T_{\text {od }}$, we also convert CFO to PO to analyze its influence and gives:

$$
\Delta \varphi_{c i}=2 \pi \Delta f_{i} T_{\mathrm{od}}
$$

where $\Delta \varphi_{c i}$ represents the PO of radio node $i$ caused by corresponding CFO $\Delta f_{i}$.

\subsection{Average Beampattern with TO and CFO}

The effects of TO and CFO on beampattern are researched respectively here. At first, we analyze the effects of TO. In presence of $\mathrm{TO}$, the radio nodes get a additional phase 
offset, and the initial phase of node $i$ in (2) can be rewritten as:

$$
\Psi_{i}=-\frac{2 \pi}{\lambda} d_{i}\left(\phi_{0}\right)+\Delta \varphi_{t i}
$$

The corresponding array factor considering PO is given by:

$$
F\left(\phi \mid \mathbf{z}, \Delta \varphi_{\mathbf{t}}\right)=\frac{1}{N} \sum_{i=1}^{N} \mathrm{e}^{-\mathrm{j} a z_{i}} \mathrm{e}^{\mathrm{j} \Delta \varphi_{t i}}
$$

where $\Delta \varphi_{\mathbf{t}}=\left[\Delta \varphi_{t 1}, \Delta \varphi_{t 2}, \cdots, \Delta \varphi_{t N}\right]^{T}$.

The far-filed beampattern is derived as:

$$
\begin{aligned}
P\left(\phi \mid \mathbf{z}, \Delta \varphi_{\mathbf{t}}\right) & =\left|F\left(\phi \mid \mathbf{z}, \Delta \varphi_{\mathbf{t}}\right)\right|^{2} \\
& =F\left(\phi \mid \mathbf{z}, \Delta \varphi_{\mathbf{t}}\right) F^{*}\left(\phi \mid \mathbf{z}, \Delta \varphi_{\mathbf{t}}\right) \\
& =\frac{1}{N}+\frac{1}{N^{2}} \sum_{i=1}^{N} \mathrm{e}^{-\mathrm{j} a z_{i}} \mathrm{e}^{\mathrm{j} \Delta \varphi_{t i}} \sum_{l=1, l \neq i}^{N} \mathrm{e}^{\mathrm{j} a z_{l}} \mathrm{e}^{-\mathrm{j} \Delta \varphi_{t l}} .
\end{aligned}
$$

The beampattern in (18) is related to random variables $z_{i}$ and $\Delta \varphi_{i}$, we still adopting the non-parametric kernel method to calculate the PDFs of $z_{i}$ and $\Delta \varphi_{i}$. Similarly with (7), we have:

$$
f_{\Delta \varphi_{t i}}(\Delta \varphi)=\frac{1}{\sqrt{2 \pi} K s} \sum_{k=1}^{K} \exp \left(-\frac{\left(\Delta \varphi-\Delta \bar{\varphi}_{t k}\right)^{2}}{2 s^{2}}\right) .
$$

Then the average beampattern of $\mathbf{z}$ and $\Delta \varphi_{\mathbf{t}}$ can be derived as:

$$
\begin{aligned}
P_{\mathrm{av}}(\phi) & =E_{\mathbf{z}, \Delta \varphi_{\mathbf{t}}}\left\{P\left(\phi \mid \mathbf{z}, \Delta \varphi_{\mathbf{t}}\right)\right\} \\
& =\frac{1}{N}+\left(1-\frac{1}{N}\right)\left|A_{z}\right|^{2}\left|A_{\Delta \varphi_{t}}\right|^{2}
\end{aligned}
$$

where $A_{\Delta \varphi_{t}}=\frac{1}{K} \sum_{k=1}^{K} \mathrm{e}^{\mathrm{j} \Delta \bar{\varphi}_{t k}}$. The detailed derivation process can be seen in Appendix. Define the additional term $\left|A_{\Delta \varphi_{t}}\right|^{2}$ as the degradation factor, and it reflects the degradation of the array gain caused by offsets.

With the similar procedure, we can get the average beampattern in presence with $\mathrm{CFO}$ as follows:

$$
F\left(\phi \mid \mathbf{z}, \Delta \varphi_{\mathbf{c}}\right)=\frac{1}{N} \sum_{i=1}^{N} \mathrm{e}^{-\mathrm{j} a z_{i}} \mathrm{e}^{\mathrm{j} \Delta \varphi_{c i}}
$$

$$
\begin{aligned}
P\left(\phi \mid \mathbf{z}, \Delta \varphi_{\mathbf{c}}\right) & =\left|F\left(\phi \mid \mathbf{z}, \Delta \varphi_{\mathbf{c}}\right)\right|^{2} \\
& =\frac{1}{N}+\frac{1}{N^{2}} \sum_{i=1}^{N} \mathrm{e}^{-\mathrm{j} a z_{i}} \mathrm{e}^{\mathrm{j} \Delta \varphi_{c i}} \sum_{l=1, l \neq i}^{N} \mathrm{e}^{\mathrm{j} a z_{l}} \mathrm{e}^{-\mathrm{j} \Delta \varphi_{c l}},
\end{aligned}
$$

$$
\begin{aligned}
P_{\mathrm{av}}(\phi) & =E_{\mathbf{z}, \Delta \varphi_{\mathbf{c}}}\left\{P\left(\phi \mid \mathbf{z}, \Delta \varphi_{\mathbf{c}}\right)\right\} \\
& =\frac{1}{N}+\left(1-\frac{1}{N}\right)\left|A_{z}\right|^{2}\left|A_{\Delta \varphi_{c}}\right|^{2}
\end{aligned}
$$

where $A_{\Delta \varphi_{c}}=\frac{1}{K} \sum_{k=1}^{K} \mathrm{e}^{\mathrm{j} \Delta \bar{\varphi}_{c k}}$ is the degradation factor caused by $\mathrm{CFO}$.

In addition to the degradation factor, we also adopt several parameters to characterize the beampattern, which are listed below:
- $3 \mathrm{~dB}$ beamwidth. The $3 \mathrm{~dB}$ beamwidth describes the width of mainlobe and is defined as the angle $\phi_{3 \mathrm{~dB}}$ at which the power of the average beampattern drops $3 \mathrm{~dB}$ below its maximum value at $\phi_{0}$.

- $3 \mathrm{~dB}$ sidelobe region. The $3 \mathrm{~dB}$ sidelobe region is defined as $S_{3 \mathrm{~dB}} \triangleq\left\{\phi\left|\phi_{\text {sidelobe }} \leq\right| \phi \mid \leq \pi\right\}$. At the angle $\phi_{\text {sidelobe }}$, the power of the average beampattern exceeds $3 \mathrm{~dB}$ above $1 / N$.

- Average directivity. The diversity is used to describe how much radiated energy is concentrated to the desired direction, relative to a single isotropic antenna. Here we adopts the normalized directivity $\tilde{D}_{\mathrm{av}}$, which is calculated as follows:

$$
\tilde{D}_{\mathrm{av}}=D_{\mathrm{av}}^{*} / N=\frac{1}{N} \frac{\int_{-\pi}^{\pi} P_{\mathrm{av}}\left(\phi_{0}\right) d \phi}{\int_{-\pi}^{\pi} P_{\mathrm{av}}(\phi) d \phi}
$$

where $D_{\mathrm{av}}^{*}$ is the average directivity.

\section{Simulation Result and Discussions}

Here we adopts the differential distribution in [9] to simulate the node distributions. Under differential distribution, the disk area with radius $R$ is divided into $L$ rings from the center to the outside. Each ring has the same width and different node densities. Nodes are randomly deployed in each ring area according to Uniform distribution. The carrier frequency of radio node is $f_{\mathrm{c}}=30 \mathrm{MHz}$. The corresponding wavelength is $\lambda=10$. Here set $R=30$ and $L=5$. The number of total radio nodes $N=16$. The node probability densities of each ring are $29.55 \%, 27.18 \%, 22.41 \%$, $15.23 \%$ and $5.63 \%$ respectively.

The DTBF in presence with TO and CFO are simulated respectively. Here two simulations are carried out to analyze the effects of offsets on beamforming performance in terms of degradation and beampattern.

Simulation 1 evaluates the accumulation effects of different TO and CFO under different observation durations. Set the observation duration $T_{\text {od }}$ as $10 \mu \mathrm{s}, 100 \mu \mathrm{s}, 200 \mu \mathrm{s}$, $500 \mu \mathrm{s}$ and $1 \mathrm{~ms}$ respectively. The frequency skew of the clock crystal $\alpha_{i}$ is assumed to follow a Gaussian distribution with mean $=1$ and standard deviation $=\rho$, where $\rho$ adopts the typical values as $1,2,5,10,20,30$, and 50 in ppm. For the $\mathrm{TO}$, the initial offset is set as a random variable on the order of nanosecond. According to (11), we got the time offsets under different durations. For the CFO, $\left\{\Delta f_{i}\right\}_{i=1, \cdots, N}$ are assigned from a Gaussian distribution with mean $=0$ and stand derivation $=\alpha_{i} f_{\mathrm{c}}$. The simulation has run 50 times. The cumulative effect of the TO and CFO in different lengths of observation duration are plotted respectively in Fig. 2 and 3. 


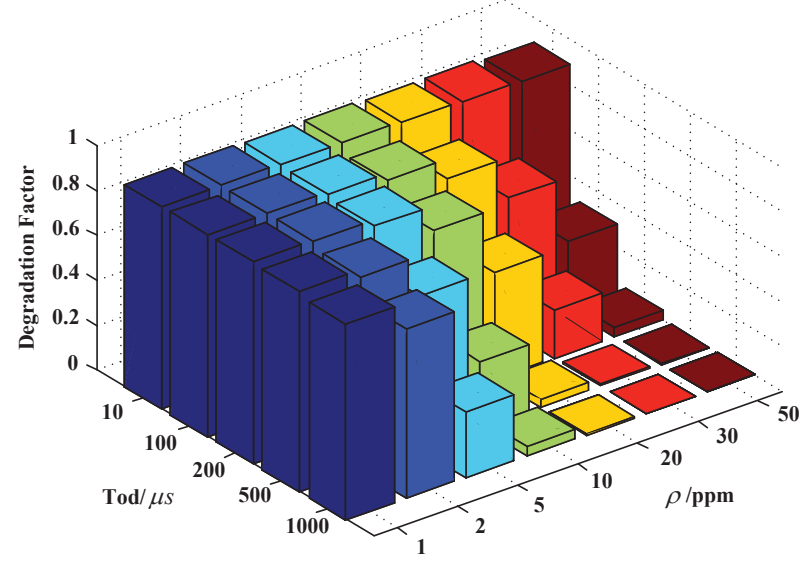

Fig. 2. Degradation comparison with TO and $f_{\mathrm{c}}=30 \mathrm{MHz}$.

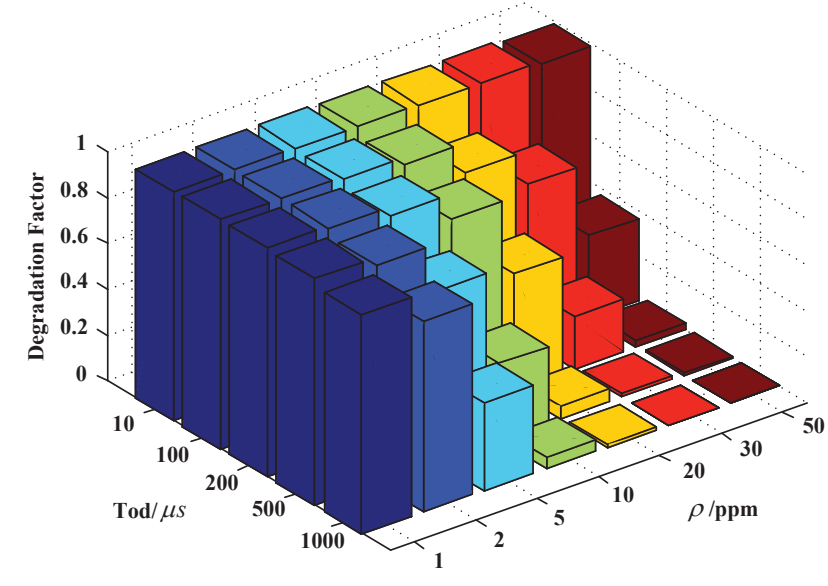

Fig. 3. Degradation comparison with $\mathrm{CFO}$ and $f_{\mathrm{c}}=30 \mathrm{MHz}$.

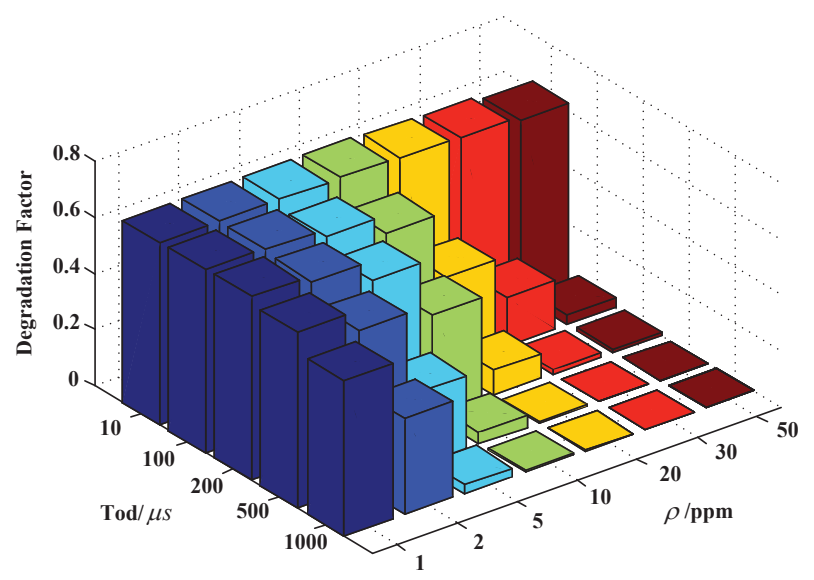

Fig. 4. Degradation comparison with TO and $f_{\mathrm{c}}=60 \mathrm{MHz}$.

The degradation factor $\left|A_{\Delta \varphi_{t}}\right|^{2}$ is related to offset. In case without offset, we have $\left|A_{\Delta \varphi_{t}}\right|^{2}=1$. This value will approach to zero as the offsets become larger. As Figures 2 and 3 show, the distributed beamformer shows

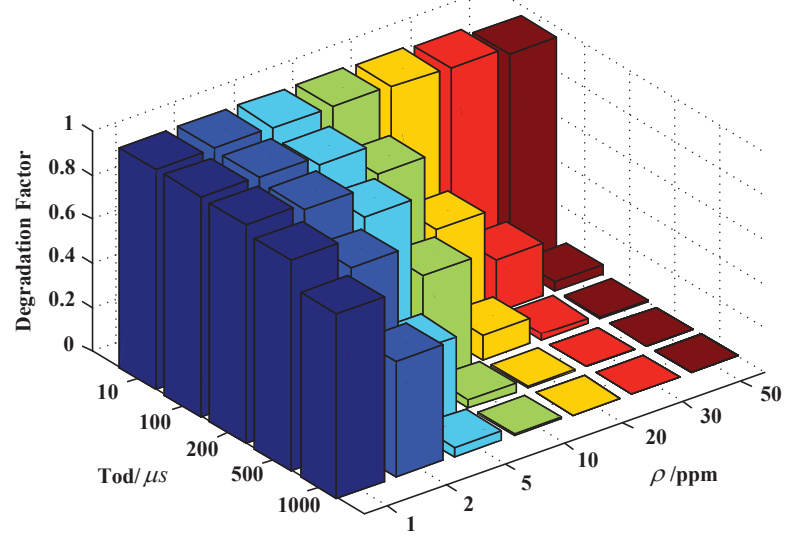

Fig. 5. Degradation comparison with $\mathrm{CFO}$ and $f_{\mathrm{c}}=60 \mathrm{MHz}$.

a certain degree of tolerance to offsets when the degradation is near 0 with a small $\rho$ and a short duration. Then with a large $\rho$ or a long duration, we may not acquire the beamforming gain due to the accumulated TO.

It is noting that $f_{\mathrm{c}}$ is introduced in the transformation of TO and CFO into PO and it also can affect the accumulation effect. Figures 4-5 show the corresponding degradation factors when $f_{\mathrm{c}}=60 \mathrm{MHz}$. We can see a larger degradation in the corresponding condition as the carrier frequency increases.

Simulation 2 tries to analyze the DTBF performance from the perspective of the beampattern characteristics. For a given observation period $T_{\text {od }}=100 \mu$ s, we consider different offsets as $\rho$ adopts 1, 10, 30, and 50 respectively. Four cases are considered here which are listed below:

Case 1: In presence with TO, and $f_{\mathrm{c}}=30 \mathrm{MHz}$.

Case2: In presence with TO, and $f_{\mathrm{c}}=60 \mathrm{MHz}$.

Case3: In presence with $\mathrm{CFO}$, and $f_{\mathrm{c}}=30 \mathrm{MHz}$.

Case4: In presence with CFO, and $f_{\mathrm{c}}=60 \mathrm{MHz}$.

Figures 6-9 show the average beampattern in presence with TO and CFO under different $f_{\mathrm{c}}$.

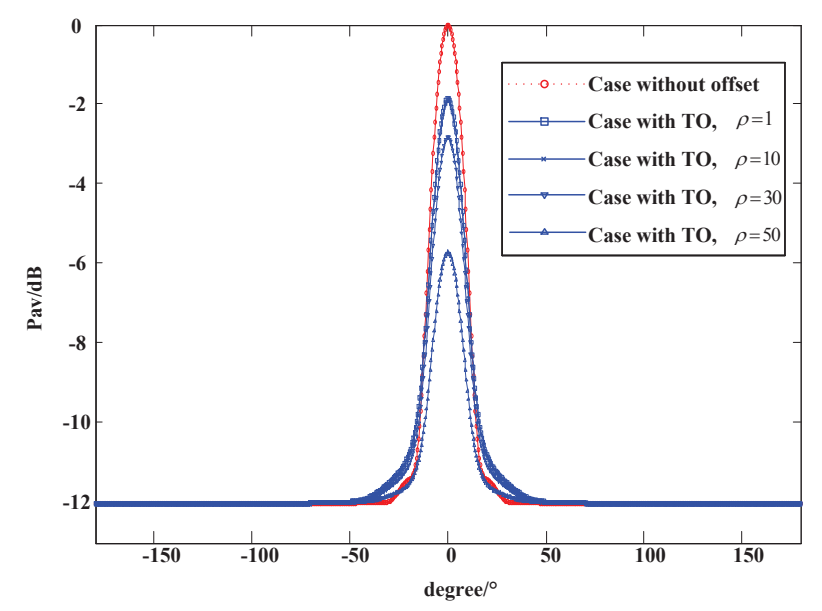

Fig. 6. Average beampattern comparison with $\mathrm{TO}$ and $f_{\mathrm{c}}=30 \mathrm{MHz}$. 


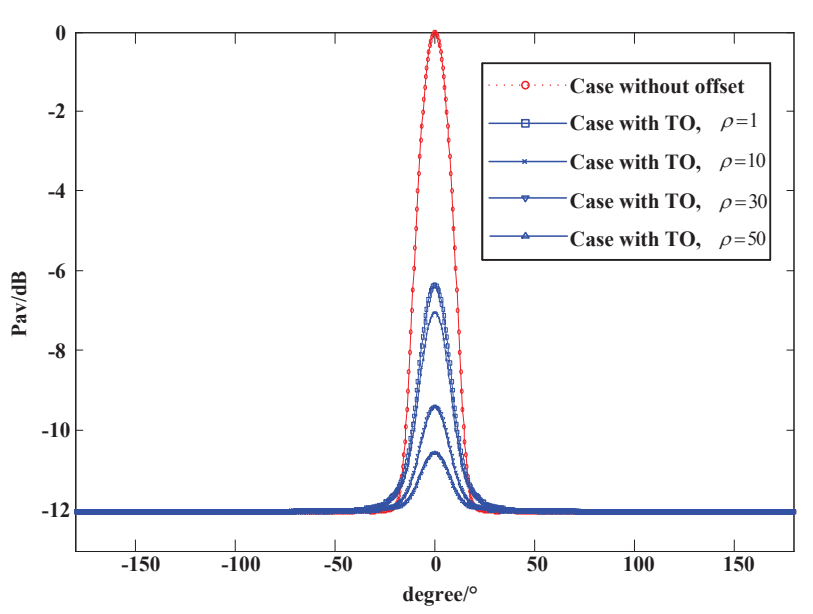

Fig. 7. Average beampattern comparison with TO and $f_{\mathrm{c}}=60 \mathrm{MHz}$

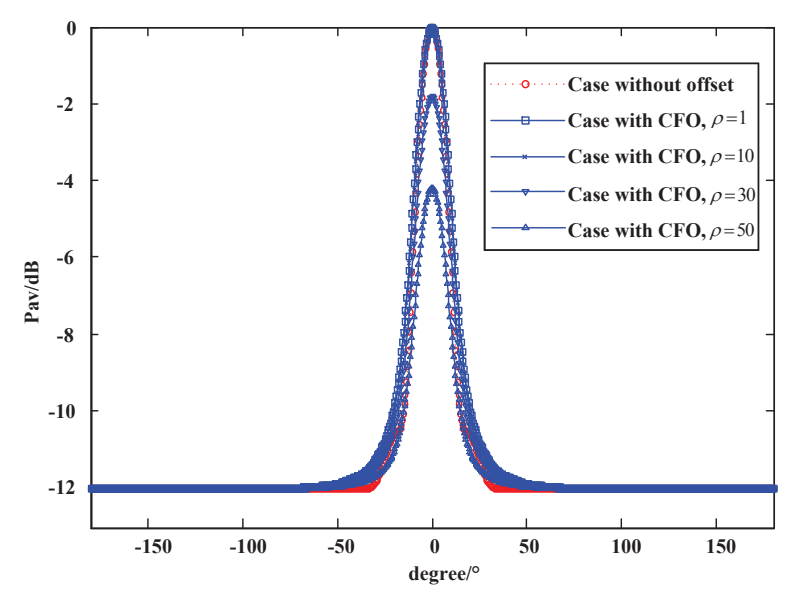

Fig. 8. Average beampattern comparison with $\mathrm{CFO}$ and $f_{\mathrm{c}}=30 \mathrm{MHz}$

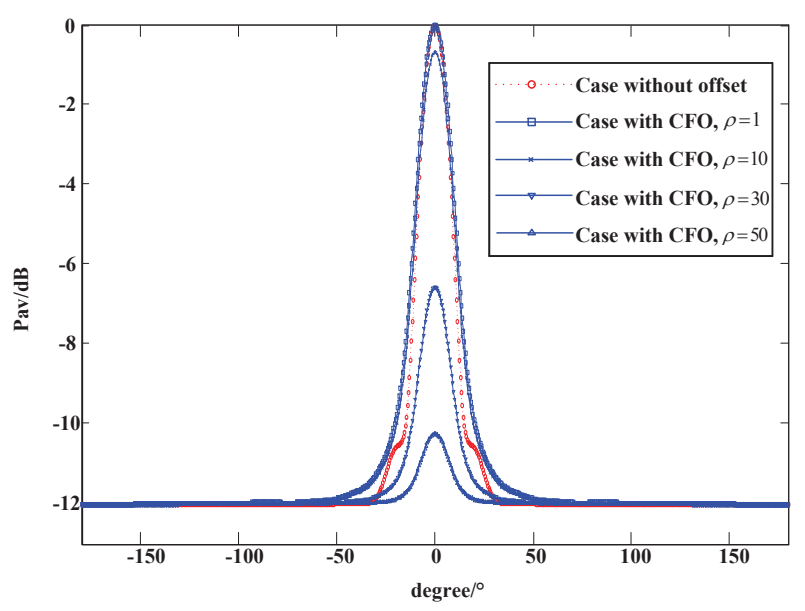

Fig. 9. Average beampattern comparison with $\mathrm{CFO}$ and $f_{\mathrm{c}}=60 \mathrm{MHz}$.

We also compare the $3 \mathrm{~dB}$ width, $3 \mathrm{~dB}$ sidelobe region and normalized directivity, which are shown in Tables 1-3, respectively.

\begin{tabular}{|c|c|c|c|c|}
\hline$\rho$ [ppm] & Case1 & Case2 & Case3 & Case4 \\
\hline 1 & 8 & 10 & 8.5 & 8.5 \\
\hline 10 & 8 & 11 & 8.5 & 8.5 \\
\hline 30 & 8 & - & 8 & 11 \\
\hline 50 & 9.5 & - & 9 & - \\
\hline
\end{tabular}

Tab. 1. The $3 \mathrm{~dB}$ width comparison.

\begin{tabular}{|c|c|c|c|c|}
\hline$\rho$ [ppm] & Case1 & Case2 & Case3 & Case4 \\
\hline 1 & 14 & 9.5 & 17.5 & 17.5 \\
\hline 10 & 14 & 8.5 & 17.5 & 16.5 \\
\hline 30 & 13 & - & 15.5 & 9.5 \\
\hline 50 & 10 & - & 12.5 & - \\
\hline
\end{tabular}

Tab. 2. The $3 \mathrm{~dB}$ sidelobe region comparison.

\begin{tabular}{|c|c|c|c|c|}
\hline$\rho$ [ppm] & Case1 & Case2 & Case3 & Case4 \\
\hline 1 & 0.4579 & 0.2216 & 0.5665 & 0.5661 \\
\hline 10 & 0.4536 & 0.2011 & 0.5552 & 0.5213 \\
\hline 30 & 0.3975 & 0.1292 & 0.4446 & 0.2077 \\
\hline 50 & 0.2472 & 0.0954 & 0.3162 & 0.1006 \\
\hline
\end{tabular}

Tab. 3. The normalized directivity comparison.

As shown in Figures 6-9, while the average power level of the sidelobe remains unchanged at $1 / N$ $\left(10 \log _{10}(1 / 16)=-12 \mathrm{~dB}\right)$, the array gain at the desired direction is pulled down because of the offsets. As $\rho$ increases, the corresponding $\mathrm{TO}$ and $\mathrm{CFO}$ increases, which leads to a larger degradation in the array gain. The three beampattern parameters in Tab. 1-3 also show the effects of TO and CFO. As $\rho$ increases, the mainlobe of the beampattern is widened, and the $3 \mathrm{~dB}$ sidelobe region is expanded. Similar conclusion can be draw from the change of $\tilde{D}_{\mathrm{av}}$ in Tab. 3. The normalized diversity decreases with increasing $\rho$, which means less powers are focused on the desired direction. For a high values of $\rho, \phi_{3 \mathrm{~dB}}$ cannot be calculated out. In some cases, $\phi_{3 \mathrm{~dB}}<\phi_{\text {sidelobe }}$ occurs, which indicates the beamformer gain cannot be guaranteed. This can be also verified by the corresponding $\tilde{D}_{\text {av }}$, which drops to around 0.1 .

As shown above, TO and CFO can degrade the DTBF performance. We need to design a periodic synchronization to deal with the effects of TO and CFO. Both the working frequency and oscillator manufactory level should be considered in order to reasonably determine the synchronization cycle. Considering the background of emergence radio, the radio node may work at the frequency hopping $(\mathrm{FH})$ mode. It is an open issue to evaluate the accommodation effects of $\mathrm{TO}$ and $\mathrm{CFO}$ as the working frequency changes.

\section{Conclusion}

This paper evaluates the effects of TO and CFO on the DTBF performance under arbitrary node distribution conditions. The analysis is carried out in statistical way. TO and $\mathrm{CFO}$ are converted to PO, and kernel method is adopted to build the PDFs of node and offsets. The average beampattern, $3 \mathrm{~dB}$ width, $3 \mathrm{~dB}$ sidelobe region and normalized directivity for different offset cases are detailed analyzed here. Theoretical analysis and simulation results show that TO and CFO can decrease the virtual array gain. This calls for a continuously 
synchronization method. Future work includes the effects analysis under frequency hopping condition and design of time and carrier frequency synchronization algorithms.

\section{Acknowledgments}

This project is supported by the National Defense Research Foundation of China.

\section{References}

[1] HER, J., WYSOKI, T. A., WYSOKI, B. J. Review of distributed beamforming. Journal of Telecommunications and Information Technology, 2011, vol. 12, no. 1, p. 78-88. ISSN: 1899-8852

[2] PREUSS, R. D., BROWN, D. R. Retrodirective distributed transmit beamforming with two-way source synchronization. In Proceedings of the 44th Annual Conference on Information Sciences and Systems (CISS). New Jersey (USA), 2010, p. 1-6. DOI: $10.1109 /$ CISS.2010.5464801

[3] BLETSAS, A., LIPPMAN, A., SAHAlOS, J. N. Simple, zero-feedback, collaborative beamforming for emergency radio. In Proceedings of the 6th International Symposium on Wireless Communication Systems. 2009, p. 657-661. DOI: 10.1109/ISWCS.2009.5285299

[4] OCHIAI, H., MITRAN, P., POOR, H. V. Collaborative beamforming for distributed wireless ad hoc sensor networks. IEEE Transactions on Signal Processing, 2005, vol. 53, no. 11, p. 4110-4124. DOI: 10.1109/TSP.2005.857028

[5] SRIPLOY, P., UTHANSAKUL, P., UTHANSAKUL, M. An effect of imperfection in node location estimation on distributed beamforming. In Proceedings of the 9th International Conference on Electrical Engineering/Electronics, Computer, Telecommunications and Information Technology (ECTI-CON). 2012, p. 1-4. DOI: 10.1109/ECTICon.2012.6254143

[6] DONG, L., PETROPUlU, A. P., POOR, H. V. Performance analysis of a cross-layer collaborative beamforming approach in the presence of channel and phase errors. In Proceedings of the IEEE International Conference on Acoustics, Speech and Signal Processing. 2008, p. 3233-3236. DOI: 10.1109/ICASSP.2008.4518339

[7] AHMED, M. F., VOROBYOV, S. A. Collaborative beamforming for wireless sensor networks with Gaussian distributed sensor nodes. IEEE Transactions on Wireless Communications, 2009, vol. 8, no. 2, p. 638-643. DOI: 10.1109/TWC.2009.071339

[8] CHEN, H. W., ZHAN, W. S., KIRING, A., et al. Distributed beamforming with uniform circular array formation in wireless sensor networks. In Proceedings of the Global High Tech Congress on Electronics. 2012, vol. 3, no. 1, p. 75-80. DOI: 10.1109/GHTCE.2012.6490128

[9] HUANG, J., WANG, P., WAN, Q. Collaborative beamforming for wireless sensor networks with arbitrary distributed sensors. IEEE Communications Letters, 2012, vol. 16, no. 7, p. 1118-1120. DOI: 10.1109/LCOMM.2012.050912.120370

[10] SHEN, X., HUANG, J., LIU, P., et al. Analysis of collaborative beamforming for wireless sensor networks with phase offset. Radioengineering, 2014, vol. 23, no. 1, p. 421-429. ISSN: 1805-9600

[11] MUDUMBAI, R., BROWN, D. R., MADHOW, U., et al. Distributed transmit beamforming: challenges and recent progress. IEEE Communications Magazine, 2009, vol. 47, no. 2, p. 102-110. DOI: 10.1109/MCOM.2009.4785387

[12] MAGGS, M. K., O'KEEFE, S. G., THIEL, D. V. Consensus clock synchronization for wireless sensor networks. IEEE Sensors Journal, 2012, vol. 12, no. 6, p. 2269-2277. DOI: 10.1109/JSEN.2011.2182045

[13] HU, P. H., TSENG, P. H., GUO, Y. Y., et al. Distributed transmit beamforming algorithms for unsynchronized OFDM systems with timing offset. IEEE Communications Letters, 2016, vol. 20, no. 9, p. 1788-1791. DOI: 10.1109/LCOMM.2016.2587774

[14] WANG, W. Q. Carrier frequency synchronization in distributed wireless sensor networks. IEEE Systems Journal, 2015, vol. 9, no. 3, p. 703-713. DOI: 10.1109/JSYST.2014.2330392

\section{About the Authors ...}

Ding YUAN (corresponding author) was born in Taian, China in July 1990. He received his B.E. degree and M.E. degree from Mechanical Engineering College respectively in 2011 and 2013. His research interests include anti-jamming technology of wireless communication.

Hou-de QUAN was born in Dalian, China in 1963. He received his $\mathrm{PhD}$. degree from Northwestern Polytechnical University, China in 2008. Now he is a professor in Dept. of Information Engineering of Mechanical Engineering College. His research interests include information and communication engineering.

Hui-xian SUN was born in Baotou, China in 1980. He received his M.E. degree and $\mathrm{PhD}$. degree from National University of Defense Technology respectively in 2005 and 2010. 


\section{Appendix}

Here we show the derivation of $P_{\text {av }}(\phi)$ in (20) in detail. According to (16), (17), (18), and (19), we have:

$$
\begin{aligned}
P_{\mathrm{av}}(\phi)= & E_{\mathbf{z}, \Delta \varphi_{\mathbf{t}}}\left\{P\left(\phi \mid \mathbf{z}, \Delta \varphi_{\mathbf{t}}\right)\right\} \\
= & \int_{-\infty}^{+\infty} \int_{-\infty}^{+\infty} P\left(\phi \mid \mathbf{z}, \Delta \varphi_{\mathbf{t}}\right) f_{z_{i}}(\mathbf{z}) f_{\Delta \varphi_{t i}}\left(\Delta \varphi_{\mathbf{t}}\right) \mathrm{d} \mathbf{z d} \Delta \varphi_{\mathbf{t}} \\
= & \int_{-\infty}^{+\infty} \int_{-\infty}^{+\infty}\left|F\left(\phi \mid \mathbf{z}, \Delta \varphi_{\mathbf{t}}\right)\right|^{2} f_{z_{i}}(\mathbf{z}) f_{\Delta \varphi_{t i}}\left(\Delta \varphi_{\mathbf{t}}\right) \mathrm{d} \mathbf{z d} \Delta \varphi_{\mathbf{t}} \\
= & \frac{1}{N}+\frac{1}{N^{2}} \sum_{i=1}^{N} \sum_{t=1}^{M} \int_{-\infty}^{+\infty} \frac{\mathrm{e}^{-\mathrm{j} a z_{i}}}{\sqrt{2 \pi} M h} \exp \left(-\frac{\left(z_{i}-\bar{z}_{t}\right)^{2}}{2 h^{2}}\right) \mathrm{d} z_{i} \\
& \cdot \sum_{k=1}^{K} \int_{-\infty}^{+\infty} \frac{\mathrm{e}^{\mathrm{j} \Delta \varphi_{t i}}}{\sqrt{2 \pi} K s} \exp \left(-\frac{\left(\Delta \varphi_{t i}-\Delta \bar{\varphi}_{t k}\right)^{2}}{2 s^{2}}\right) \mathrm{d} \Delta \varphi_{t i} \\
& \cdot \sum_{l=1, l \neq i}^{N} \sum_{t=1}^{M} \int_{-\infty}^{+\infty} \frac{\mathrm{e}^{\mathrm{j} a z_{l}}}{\sqrt{2 \pi} M h} \exp \left(-\frac{\left(z_{l}-\bar{z}_{t}\right)^{2}}{2 h^{2}}\right) \mathrm{d} z_{l} \\
& \cdot \sum_{k=1}^{K} \int_{-\infty}^{+\infty} \frac{\mathrm{e}^{-\mathrm{j} \Delta \varphi_{t l}}}{\sqrt{2 \pi} K s} \exp \left(-\frac{\left(\Delta \varphi_{t l}-\Delta \bar{\varphi}_{t k}\right)^{2}}{2 s^{2}}\right) \mathrm{d} \Delta \varphi_{t l}
\end{aligned}
$$

where

$$
\begin{aligned}
& \int_{-\infty}^{+\infty} \frac{\mathrm{e}^{\mathrm{j} \Delta \varphi_{t i}}}{\sqrt{2 \pi} K s} \exp \left(-\frac{\left(\Delta \varphi_{t i}-\Delta \bar{\varphi}_{t k}\right)^{2}}{2 s^{2}}\right) \mathrm{d} \Delta \varphi_{t i} \\
& =\frac{1}{K} \exp \left(\frac{\left(j s^{2}+\Delta \varphi_{t k}\right)^{2}-\Delta \varphi_{t k}^{2}}{2 s^{2}}\right) \\
& \cdot \int_{-\infty}^{+\infty} \frac{1}{\sqrt{2 \pi} s} \exp \left(-\frac{\left(\Delta \varphi_{t i}-\left(\Delta \varphi_{t k}+j s^{2}\right)\right)^{2}}{2 s^{2}}\right) \mathrm{d} \Delta \varphi_{t i}
\end{aligned}
$$

According to the theory of kernel method, the second term in (26) is equal to 1 . Then (26) can be derived as:

$$
\begin{array}{r}
\int_{-\infty}^{+\infty} \frac{\mathrm{e}^{\mathrm{j} \Delta \varphi_{t i}}}{\sqrt{2 \pi} K s} \exp \left(-\frac{\left(\Delta \varphi_{t i}-\Delta \bar{\varphi}_{t k}\right)^{2}}{2 s^{2}}\right) \mathrm{d} \Delta \varphi_{t i} \\
=\frac{1}{K} \mathrm{e}^{-\frac{s^{2}}{2}} \mathrm{e}^{\mathrm{j} \Delta \bar{\varphi}_{t k}} .
\end{array}
$$

With similar derivation, we have:

$$
\begin{aligned}
& \int_{-\infty}^{+\infty} \frac{\mathrm{e}^{-\mathrm{j} a z_{i}}}{\sqrt{2 \pi} M h} \exp \left(-\frac{\left(z_{i}-\bar{z}_{t}\right)^{2}}{2 h^{2}}\right) \mathrm{d} z_{i} \\
& =\frac{1}{M} e^{-\frac{a^{2} h^{2}}{2}} \mathrm{e}^{-\mathrm{j} a \bar{z}_{t}}, \\
& \int_{-\infty}^{+\infty} \frac{\mathrm{e}^{-\mathrm{j} \Delta \varphi_{t l}}}{\sqrt{2 \pi} K s} \exp \left(-\frac{\left(\Delta \varphi_{t l}-\Delta \bar{\varphi}_{t k}\right)^{2}}{2 s^{2}}\right) \mathrm{d} \Delta \varphi_{t l} \\
& =\frac{1}{K} \mathrm{e}^{-\frac{s^{2}}{2}} \mathrm{e}^{-\mathrm{j} \Delta \bar{\varphi}_{t k}}, \\
& \int_{-\infty}^{+\infty} \frac{\mathrm{e}^{\mathrm{j} a z_{l}}}{\sqrt{2 \pi} M h} \exp \left(-\frac{\left(z_{l}-\bar{z}_{t}\right)^{2}}{2 h^{2}}\right) \mathrm{d} z_{l} \\
& =\frac{1}{M} \mathrm{e}^{-\frac{a^{2} h^{2}}{2}} \mathrm{e}^{\mathrm{j} a \bar{z}_{t}} .
\end{aligned}
$$

Substituting (27), (28), (29), and (30) into (25), we have:

$$
\begin{aligned}
P_{\text {av }}(\phi)= & \frac{1}{N}+\frac{1}{N^{2}} \sum_{i=1}^{N} \sum_{t=1}^{M} \frac{1}{M} \mathrm{e}^{-\frac{a^{2} h^{2}}{2}} \mathrm{e}^{-\mathrm{j} a \bar{z}_{t}} \sum_{k=1}^{K} \frac{1}{K} \mathrm{e}^{-\frac{s^{2}}{2}} \mathrm{e}^{\mathrm{j} \Delta \bar{\varphi}_{t k}} \\
& \cdot \sum_{l=1, l \neq i}^{N} \sum_{t=1}^{M} \frac{1}{M} \mathrm{e}^{-\frac{a^{2} h^{2}}{2}} \mathrm{e}^{-\mathrm{j} a \bar{z}_{t}} \sum_{k=1}^{K} \frac{1}{K} \mathrm{e}^{-\frac{s^{2}}{2}} \mathrm{e}^{-\mathrm{j} \Delta \bar{\varphi}_{t k}} \\
= & \frac{1}{N}+\left(1-\frac{1}{N}\right) \mathrm{e}^{-s^{2}} \mathrm{e}^{-a^{2} h^{2}}\left|A_{z}\right|^{2}\left|A_{\Delta \varphi_{t}}\right|^{2}
\end{aligned}
$$

where $A_{\Delta \varphi_{t}}=\frac{1}{K} \sum_{k=1}^{K} \mathrm{e}^{\mathrm{j} \Delta \bar{\varphi}_{t k}}$ and $A_{z}=\frac{1}{M} \sum_{t=1}^{M} \mathrm{e}^{-\mathrm{j} a \bar{z}_{t}}$.

When the size of the survey set is large enough, $P_{\mathrm{av}}(\phi)$ can be described as:

$$
\begin{aligned}
P_{\mathrm{av}}(\phi) & =E_{\mathbf{z}, \Delta \varphi_{\mathbf{t}}}\left\{P\left(\phi \mid \mathbf{z}, \Delta \varphi_{\mathbf{t}}\right)\right\} \\
& =\frac{1}{N}+\left(1-\frac{1}{N}\right)\left|A_{z}\right|^{2}\left|A_{\Delta \varphi_{t}}\right|^{2} .
\end{aligned}
$$

As [9] shown, the size of the survey set can affect accurate of the kernel method. With enough $\mathrm{M}$ and $\mathrm{K}$, we can get the stable values of the estimated parameters.

When $\Delta \varphi_{\mathrm{t}}$ is replaced by $\Delta \varphi_{\mathbf{c}}$, we can get $P_{\mathrm{av}}(\phi)$ in (23) with similar derivation as shown above. 